\title{
Special issue for COMPSE 2016
}

\section{First EAl international Conference on computer science and Engineering, November 11-12, 2016, Penang, Malaysia}

\author{
Pandian Vasant ${ }^{1} \cdot$ Gerhard-Wilhelm Weber ${ }^{2,3} \cdot$ Vinh T. Le $^{4}$
}

Published online: 13 January 2018

๑) Springer-Verlag GmbH Germany, part of Springer Nature 2018

In recent years, modern Operational Research (OR) and Optimization approaches have gained an increasing number of researchers, educators, decision makers and practitioners. In fact, very powerful and intelligent ("smart") computational technologies emerged for coping with a vast number of real-life tasks and challenges, especially, related to the fields of optimization in theory and application. These challenges are characterized through their extremely high complexity. In the families of international OR and Analytics and of Computation, scholars from numerous sectors, spectrums and countries have collaborated on solutions for those emerging problems. Inside that scientific community there are scientists from IFORS and its continental groupings and, within them, national OR societies and working groups.

The high world population which increased so strongly in recent decades, the feeling of loneliness of many humans, of difficulties with the rapid speed of the process of "globalization" almost everywhere in the world, the related diseases, societal crises and conflicts on the globe, requires that the "Human Factor" should be taken into account much more

Pandian Vasant

pvasant@gmail.com

Gerhard-Wilhelm Weber

gerhard.weber@put.poznan.pl

Vinh T. Le

lethevinh@tdt.edu.vn

1 Faculty of Science and Information Technology, Universiti Teknologi PETRONAS, Seri Iskandar, Malaysia

2 Faculty of Engineering Management, Poznan University of Technology, Poznan, Poland

3 Institute of Applied Mathematics, METU, Ankara, Turkey

4 Faculty of Electrical and Electronics Engineering, Ton Duc Thang University, Ho Chi Minh City, Vietnam than in the past by scientists, teachers, managers, politicians, engineers, and even medical doctors, consultants and therapists.

These important expressions, considerations and implications of Intelligence, of Humility and the domains of the Humanities therefore ought to enter more and more the domains of OR, of Analytics and of Computation.

In the presence of an environment full of turbulences, classical and traditional approaches are struggling to obtain "holistic" answers and solutions with a high-level of satisfaction and fulfillment in real-world application contexts of wider OR. Therefore, new "global" optimization and analytic technologies are needed to seriously cope with these issues. One class of such methods are modern optimization techniques which encompass a generic, flexible, robust and versatile framework to manage and solve challenges of complexity in monitoring and optimizing, in controlling, fostering and guiding real-life applications of economies and financial sectors, of natural environments and high-tech, of politics and, last but not least, the well-being of humans, of social entities and communities.

This special issue of Journal of Ambient Intelligence and Humanized Computing offers new approaches of modeling and solution, and it may become a precious compendium for students, both graduate and postgraduate, for deciders and investigators in private fields and sectors, at universities and in emerging industries, related with a variety of sciences, high-tech and managerial areas, such as applied mathematics, economics, OR, game theory, physics, chemistry, computer science, environmental sciences, geo-, neuro- and bio-sciences, the humanities, societal life and developmental studies, whenever humans wish or urgently needs to model and overcome real-world challenges under uncertainty and in the presence of the "human factor".

In this context, our special issue wishes to offer a significance and to become help- and fruitful for the people 
on earth, their health, dignity, integrity, living-conditions and peace.

Subsequently please find a short introduction of the articles of this novel and pioneering issue of premium Journal of Ambient Intelligence and Humanized Computing.

1. Industry 4.0 framework for management and operations: a review

Development of markets and customer requirements with highest level of precision has been achieved created with technology and information systems, a new way of make the operations in the companies, and just the companies with the ability to adapt faster to technological innovations may remain on the market. The last industrial revolution, known as industry 4.0 perceives the operations as a holistic system, its represents a challenge that must be fulfilled and faced in order to achieve stability and permanence in the market, from the viewpoint of our world economies. In the organizational and business area, all operations have to be linked to computer systems and management of information within the network, causing greater efficiency inside of the flow. With this new perception of industry and business, it involves different analytical tools which aim at higher efficiency in the service to the consumers, resulting in a higher competitiveness in the market and in making a difference. This article is one of the first surveys providing an extensive analysis and review of 110 publications which appeared from $1 / 01 / 12$ to $20 / 02 / 17$. The goal of the study is to categorically analyze recent advances through qualitative and segmentation methods, allowing for revealing trends and areas of opportunity in the industry sense of 4.0, to cover research gaps that may be executed in organizational systems on the value chain. Another purpose is to be a reference for future research related to the categories selected in this article.

2. An optimal text compression algorithm based on frequent pattern mining

Data compression as a research area has been explored in depth over the years, resulting in Huffman Encoding, LZ77, LZW, GZip, RAR, etc. The research has widely focused on conventional character/word based mechanism without looking from the larger perspective of pattern retrieval from dense and large datasets. The authors explore the compression perspective of Data Mining suggested by Naren Ramakrishnan et al., where in Huffman Encoding there is enhanced through frequent pattern mining (FPM) a nontrivial phase in Association Rule Mining (ARM) technique. This article proposes a novel frequent pattern mining based Huffman Encoding algorithm for textual data and employs a Hash table in the process of counting of frequent patterns.
The proposed algorithm operates on a pruned set of frequent patterns, and it is also efficient in terms of database scan and storage space by reducing the code table size. Optimal (pruned) set of patterns is employed in the encoding process instead of character-based approach of conventional Huffman Encoding. Simulation results over 18 benchmark corpora demonstrate an improvement in compression ratio ranging from $18.49 \%$ over sparse datasets to $751 \%$ over dense datasets. It is also demonstrated that the proposed algorithm achieves a pattern space reduction ranging from $5 \%$ over sparse datasets to $502 \%$ in dense corpus.

3. Evaluation of inequality and technical efficiency of federal health financing for population without social security per Federal Entity, 2004-2012 in México

The main goal of this article is evaluate inequality and technical efficiency of federal health financing for population without social security per federative entity. The authors estimated two inequality measures of federal financing for population without social security per Federal Entity: Gini coefficient and Theil entropy index. Four stochastic frontier models were calculated to measure technical efficiency of health production per federative entity (2004-2012). Information on health expenditure, physical and human resources provided by the Ministry of Health through SINAIS was used. The federal financing for population without social security has reduced inequality among Federal Entities due to the incorporation of the SPSS (Mexican system of social protection in terms of health). The estimate of technical efficiency of Federal Entities through stochastic frontiers shows that most of these entities have health production inefficiencies both at outpatient and hospital levels, the outpatient level being the one with higher inefficiency. Not necessarily entities that receive greater resources produce more health. The existence of multiple financing sources has limited the effect of the SPSS to reduce the inequality in financing for the population which has no social security among Federal Entities. More health resources are needed in order to face demographic and epidemiological transitions. However, it is necessary to spend the available resources in a more efficient way. There are three main lines of action in terms of financing: structuring financing sources, improving the allocation mechanisms, and strengthening evaluation and monitoring resources exercise.

4. The urban transport planning with uncertainty in demand and travel time: a comparison of two defuzzification methods

Nowadays, the traffic congestion is a common problem in major cities; every time the travel time is increasing and also the number of private cars. It is urgent to take actions to 
solve this problem. The urban transport is becoming into the best way to combat congestion; but to make it more attractive to users it has to be more efficient (less travel time, less waiting time, low fare). The urban transport process has four main activities: network design, timetabling, vehicle scheduling and crew scheduling. The problem presented here is about the integration of the frequency and departure time scheduled both are sub activities of the timetable construction, besides it includes multiple periods planning and multi-period synchronization; therefore, the authors consider uncertainty in demand and travel time using fuzzy numbers. The planners have faced this problem every day. The authors created a mathematical model including the characteristics previously mentioned, the objectives of this model are to minimize the total operation cost, to maximize the number of multi-period synchronization between routes, and to minimize the total waiting time for passengers. The SAUGMECON method is used to solve the problem, 32 instances were randomly generated based on real data, and the comparison of two defuzzification methods ( $\mathrm{k}$-preference and second index of Yager) is presented. Finally, a comparison of the problems with uncertainty on demand and uncertainty on travel time is presented.

5. Research and development project portfolio selection under uncertainty

The problem of project portfolio selection consists in allocating resources (e.g., money) to a set of proposals optimizing certain impact measures. The authors of this article build a model which takes into account the following characteristics: projects tasks, different resources allocation policies, interdependence between tasks and/or projects, portfolio balancing rules, uncertainty in the overall budget, and uncertainty in the amount of resources requested by tasks. Uncertain parameters are represented as fuzzy triangular numbers, and fuzzy programming is employed for solving the model under uncertainty. Computational results are provided for medium- and large-scale instances.

\section{Lead time performance in an internet product delivery supply chain with automatic consolidation}

Internet sales have increased exponentially in the last decade. Much of the internet sales are of physical products in urban areas that require product delivery transportation with a tight delivery lead time. With this challenge, a new type of transportation services has been developed to cope with a strict control of transportation lead time. In this article, an internet product delivery service with customer orders which are multi-item as well as single-item is simulated. The authors specifically address the mismatch between supply and demand when retailers for any reason are unable to estimate the configuration of multi-item orders. Three scenarios of demand patterns are simulated (demand as forecasted, lower than forecasted, and higher than forecasted) using discrete-event simulation to look at the effect on transportation lead time. The results obtained show a positive effect on the mismatch between demand and resource capacity which is expressed in a higher number of delayed delivery orders. The excess of capacity in the product delivery supply chain does not have a positive impact on delivery time of orders, since technically orders are not delivered before the multi-item components are not available. This leads to think that the excess of resources is not an element that adds value to customers waiting for their orders.

\section{Agile risk management using software agents}

Risk management is an important process in Software Engineering. However, it can be perceived as somewhat contrary to the more lightweight processes used in Agile methods. Thus, an appropriate and realistic risk management model is required as well as a tool support which minimizes human effort. The authors propose the use of software agents to carry out risk management tasks and employ data collected from the project environment to detect risks. This article describes the underlying risk management model in an agile risk tool where software agents are used to support identification, assessment and monitoring of risk. It demonstrates the interaction between agents, agents' compliance with designated rules, and how they can react to changes in project-environment data. The results, demonstrated in the form of case studies, show that agents are of use for detecting risk and reacting dynamically to changes in project environment. Thus, this study can help to minimize the human effort in managing risk.

\section{Volunteer selection based on crowdsourcing approach}

Voluntary work is important today's world. There are various versions of the volunteer management system referenced in industry resources. Several organizations have developed volunteer management systems designed to incorporate spontaneous volunteers. However, it can be hard to find and recruit suitable volunteers for volunteer organizations, since the volunteers have to match many criteria with tasks. Furthermore, there is still a lack of information on the process of crowdsourcing in volunteering perspective. In this article, the authors conduct a review of volunteering management systems and crowdsourcing approach. Based on the insights derived from this analysis, they identify some issues for future research. To solve this problem, they designed a framework for the crowdsourcing approach in volunteering system to automate the process of selection volunteers and match with the criteria of volunteers and 
tasks. Crowdsourcing is one of the best approaches to get more information and faster from the crowd and to be more precise with the requirement from beneficiaries. Fuzzy systems are suitable for such decision-making environments. The implications of the findings for volunteering system are discussed, and future research directions suggested.

9. Fast marching method and modified features fusion in enhanced dynamic hand gesture segmentation and detection method under complicated background

Recent development in the field of human-computer interaction has led renewed interest in dynamic hand gesture segmentation based on gesture recognition system. Despite its long clinical success, dynamic hand gesture segmentation using webcam vision becomes technically challenging and suffers from the problem of non-accurate and poor hand gesture segmentation where the hand region is not integral due to complicated environment, partial occlusion and light effects. Therefore, for segmenting complete hand gesture region and improving the segmentation accuracy, this article proposes a combination of four modified visual features segmentation procedures, which are skin, motion, skin moving as well as contour features and fast marching method. Quantitative measurement was performed for evaluating hand gesture segmentation algorithm. Besides, qualitative measurement was done to conduct a comparison based on segmentation accuracy with previous studies. Consequently, the experiment results showed a great enhancement in hand area segmentation with a high accuracy rate of $98 \%$.

We, the Guest Editors, we would like to express our sincere thanks to all the authors for the valuable contributions and to the reviewers for their important service, care and helpful comments. Eventually, we are very grateful to the Editor-in-Chief Prof. Dr. Vincenzo Loia of Journal of Ambient Intelligence and Humanized Computing, a journal of excellence, for having given us the opportunity of preparing this special issue, and to the coworkers of Springer Verlag who were with us and supported us at every stage of preparing this special issue.

\section{References}

Arratia-Martinez NM, Caballero-Fernandez R, Litvinchev I et al (2017) Research and development project portfolio selection under uncertainty. J Ambient Intell Human Comput. https://doi. org/10.1007/s12652-017-0564-7

Avila-Torres P, Caballero R, Litvinchev I et al (2017) The urban transport planning with uncertainty in demand and travel time: a comparison of two defuzzification methods. J Ambient Intell Human Comput. https://doi.org/10.1007/s12652-017-0545-x

Cruz-Mejia O, Marmolejo JA, Vasant P (2017) Lead time performance in a internet product delivery supply chain with automatic consolidation. J Ambient Intell Human Comput. https://doi.org/10.1007/ s12652-017-0577-2

Mazlan N, Syed Ahmad SS, Kamalrudin M (2017) Volunteer selection based on crowdsourcing approach. J Ambient Intell Human Comput. https://doi.org/10.1007/s12652-017-0490-8

Odzaly EE, Greer D, Stewart D (2017) Agile risk management using software agents. J Ambient Intell Human Comput. https://doi. org/10.1007/s12652-017-0488-2

Oswald C, Sivaselvan B (2017) An optimal text compression algorithm based on frequent pattern mining. J Ambient Intell Human Comput. https://doi.org/10.1007/s12652-017-0540-2

Rodríguez-Aguilar R, Marmolejo-Saucedo JA, Vasant P (2017) Evaluation of inequality and technical efficiency of federal health financing for population without social security per Federal Entity, 2004-2012 in México. J Ambient Intell Human Comput. https:// doi.org/10.1007/s12652-017-0532-2

Saucedo-Martínez JA, Pérez-Lara M, Marmolejo-Saucedo JA et al (2017) Industry 4.0 framework for management and operations: a review. J Ambient Intell Human Comput. https://doi.org/10.1007/ s12652-017-0533-1

Thabet E, Khalid F, Sulaiman PS et al (2017) Fast marching method and modified features fusion in enhanced dynamic hand gesture segmentation and detection method under complicated background. J Ambient Intell Human Comput. https://doi.org/10.1007/ s12652-017-0512-6 